\begin{tabular}{|c|l|}
\hline Title & Singularities of lightlike hypersurfaces in Minkowski four-space \\
\hline Author(s) & Izumiya, Shyuichi; Kossowski, Marek; Pei, Donghe; Romero Fuster, M. Carmen \\
\hline Citation & $\begin{array}{l}\text { Tohoku Mathematical Journal, 58(1), 71-88 } \\
\text { https://doi.org/10.2748/tmj/1145390206 }\end{array}$ \\
\hline Issue Date & 2006-03 \\
\hline Doc URL & http://hdl.handle.net/2115/17260 \\
\hline Rights & Copyright $\odot 2006$ by THE TOHOKU UNIVERSITY \\
\hline Type & article (author version) \\
\hline File Information & TMJ58-1.pdf \\
\hline
\end{tabular}

Instructions for use 


\title{
Singularities of lightlike hypersurfaces in Minkowski four-space
}

\author{
Shyuichi Izumiya*, Marek Kossowski, Donghe Pei ${ }^{\dagger}$ \\ AND \\ M. Carmen Romero Fuster ${ }^{\ddagger}$
}

\begin{abstract}
We classify singularities of lightlike hypersurfaces in Minkowski 4-space via the contact invariants for the corresponding spacelike surfaces and lightcones.
\end{abstract}

\section{Introduction}

The objective of this paper (and [5-9]) is to link the differential geometry of lightlike hypersurfaces in Minkowski 4-space with the modern theory of Legendrian singularities. Lightlike hypersurfaces are ruled 3-manifolds whose induced first fundamental forms are positive semi definite. Extending these ruling lines defines a natural completion which contains (nonimmersive) singular points. The generic intersection of such a hypersurface with a spacelike 3-plane is an immersed 2-manifold which encodes the local differential geometry of lightlike hypersurfaces $[9,10]$. However, this approach does not efficiently adapt to more general spacetimes. As an alternative we will use Montaldi's characterization of submanifold contacts in terms of $\mathcal{K}$-equivalent functions, which provides a technical linkage to Legendrian singularity theory. As a consequence, we provide a local classification of lightlike hypersurface singularities in terms of algebraic invariants (an $\mathbb{R}$-algebra) and differential geometric invariants (the lightcone indicatrix). In [3, 4] lightlike hypersurfaces have been studied from the viewpoint of the general theory of relativity. In this

2000 Mathematics Subject classification. Primary 53A35; Secondary 58C27

Key words and phrases. Lightlike hypersurface, spacelike surface, lightcone, Lorentzian distancesquared function

* partially supported by Grant-in-Aid for formation of COE

$\dagger$ partially supported by NSF of China No. 10471020

$\ddagger$ partially supported by DGCYT grant No. BFM2000-1110 
paper we study the detailed differential geometric properties of lightlike hypersurfaces (and corresponding spacelike surfaces).

In Section 2 we begin by describing Cartan's frame method adapted to spacelike surfaces as well as lightlike hypersurfaces (See [7] for a more detailed discussion.) This is used to define the lightcone indicatrix. In Section 3 we describe the (multivalued) Legendrian distance squared function whose discriminant is a given lightlike hypersurface. The given hypersurface is now the wave front set of this function, as described in Legendrian singularity theory [1]. Section 4 applies Montaldi's theorem to the description of generic contact between a given lightcone and a spacelike surface. Singularities in the hypersurface are now characterized as points of higher order contact. We can also consider the contact of spacelike surfaces with other pseudo-spheres (i.e. hyperbolic spaces or de Sitter spaces). However the most interesting case is to consider the contact with lightcones. Moreover, from the point of view of physics, lightlike hypersurfaces are of importance because they are models of different types of horizons studied in relativity theory $[2,14]$. Therefore we only consider the singularities of lightlike hypersurfaces in this paper. In Section 5 we present the classification of lightlike hypersurface singularities and tangent lightcone indicatrices, which is based on the theory of Legendrian singularities [1, 19]. (See the appendix for a brief description). As a source of examples and motivation, Section 6 indicates that generic lightlike hypersurface singularities occur in the the level surfaces of solutions to the eikonal PDE on Minkowski 4-space. Section 7 indicates how these methods can be locally adopted to some curved spacetimes. Finally, we remark that many arguments in this paper can be directly generalized to higher dimensional Minkowski spaces. However, from the viewpoint of physics, Minkowski 4-space (i.e. space-time) is the most important and we need much more pages for writing the higher dimensional cases, so that we only consider 4-dimensional Minkowski space here.

We assume throughout the paper that all manifolds and maps are $C^{\infty}$ unless otherwise stated.

\section{Local differential geometry of spacelike surfaces}

In [7] we introduced the basic geometric tools for the study of spacelike surfaces in Minkowski 4-space. Here we briefly review a part of the theory relevant to this paper.

Let $\mathbb{R}^{4}=\left\{\left(x_{1}, x_{2}, x_{3}, x_{4}\right) \mid x_{1}, x_{2}, x_{3}, x_{4} \in \mathbb{R}\right\}$ be a cartesian 4-space. For any vectors $\boldsymbol{x}=\left(x_{1}, x_{2}, x_{3}, x_{4}\right), \boldsymbol{y}=\left(y_{1}, y_{2}, y_{3}, y_{4}\right)$ in $\mathbb{R}^{4}$, the pseudoscalar product of $\boldsymbol{x}$ and $\boldsymbol{y}$ is defined by $\langle\boldsymbol{x}, \boldsymbol{y}\rangle=-x_{1} y_{1}+x_{2} y_{2}+x_{3} y_{3}+x_{4} y_{4}$. We call $\left(\mathbb{R}^{4},\langle\rangle,\right)$ a Minkowski 4 -space and simply write it as $\mathbb{R}_{1}^{4}$ instead of $\left(\mathbb{R}^{4},\langle\rangle,\right)$.

We say that a vector $\boldsymbol{x}$ in $\mathbb{R}_{1}^{4} \backslash\{0\}$ is spacelike, lightlike or timelike if $\langle\boldsymbol{x}, \boldsymbol{x}\rangle>0,=0$ 
or $<0$, respectively. The norm of the vector $\boldsymbol{x} \in \mathbb{R}_{1}^{4}$ is defined by $\|\boldsymbol{x}\|=\sqrt{|\langle\boldsymbol{x}, \boldsymbol{x}\rangle|}$.

Let $\boldsymbol{X}: U \rightarrow \mathbb{R}_{1}^{4}$ be a regular surface (i.e. an immersion), where $U \subset \mathbb{R}^{2}$ is an open subset. We identify $M=\boldsymbol{X}(U)$ with $U$ through the immersion $\boldsymbol{X}$.

We call $M$ a spacelike surface if the tangent plane $T_{p} M$ of $M$ is a spacelike plane (i.e. consists of spacelike vectors) for any point $p \in M$. In this case, the normal space $N_{p} M$ is a timelike plane (i.e. Lorentz plane) (cf.[17]). Let $\left\{\boldsymbol{e}_{3}(x, y), \boldsymbol{e}_{4}(x, y)\right\}$ be an orthonormal frame of $T_{p} M$ and $\left\{\boldsymbol{e}_{1}(x, y), \boldsymbol{e}_{2}(x, y)\right\}$ a pseudo-orthonormal frame of $N_{p} M$, where $p=\boldsymbol{X}(x, y)$. Here, $\boldsymbol{e}_{1}(p)$ is a timelike vector and $\boldsymbol{e}_{i}, i=2,3,4$, are spacelike vectors.

In order to establish the fundamental formula for a spacelike surface in $\mathbb{R}_{1}^{4}$, we define some notions similar to those of Little [11]. As usual, define the forms $\omega_{i}=\delta\left(\boldsymbol{e}_{i}\right)\left\langle d \boldsymbol{X}, \boldsymbol{e}_{i}\right\rangle$ and $\omega_{i j}=\delta\left(\boldsymbol{e}_{j}\right)\left\langle d \boldsymbol{e}_{i}, \boldsymbol{e}_{j}\right\rangle$, where

$$
\delta\left(\boldsymbol{e}_{i}\right)=\operatorname{Sign}\left(\boldsymbol{e}_{i}\right)= \begin{cases}1, & i=2,3,4, \\ -1, & i=1 .\end{cases}
$$

Here $\left\langle d \boldsymbol{X}, \boldsymbol{e}_{j}\right\rangle$ denotes the scalar product of the vector valued one-form $d \boldsymbol{X}$ and the vector $\boldsymbol{e}_{j}$. Then we have $d \boldsymbol{X}=\sum_{i=1}^{4} \omega_{i} \boldsymbol{e}_{i}$ and $d \boldsymbol{e}_{i}=\sum_{j=1}^{4} \omega_{i j} \boldsymbol{e}_{j}, i=1,2,3,4$. We have the Codazzi type equations:

$$
\left\{\begin{array}{l}
d \omega_{i}=\sum_{j=1}^{4} \delta\left(\boldsymbol{e}_{i}\right) \delta\left(\boldsymbol{e}_{j}\right) \omega_{i j} \wedge \omega_{j} \\
d \omega_{i j}=\sum_{k=1}^{4} \omega_{i k} \wedge \omega_{k j}
\end{array}\right.
$$

where $d$ denotes exterior differentiation. Also, we have

$$
\omega_{i j}=-\delta\left(\boldsymbol{e}_{i}\right) \delta\left(\boldsymbol{e}_{j}\right) \omega_{j i} .
$$

In particular, $\omega_{i i}=0$ for $i=1,2,3,4$.

It follows from the fact $\left\langle d \boldsymbol{X}, \boldsymbol{e}_{1}\right\rangle=\left\langle d \boldsymbol{X}, \boldsymbol{e}_{2}\right\rangle=0$ that

$$
\omega_{1}=\omega_{2}=0 .
$$

Therefore we have

$$
\left\{\begin{array}{l}
0=d \omega_{1}=\sum_{j=1}^{4} \delta\left(\boldsymbol{e}_{1}\right) \delta\left(\boldsymbol{e}_{j}\right) \omega_{1 j} \wedge \omega_{j}=-\sum_{j=3}^{4} \delta\left(\boldsymbol{e}_{j}\right) \omega_{1 j} \wedge \omega_{j}=-\omega_{13} \wedge \omega_{3}-\omega_{14} \wedge \omega_{4}, \\
0=d \omega_{2}=\sum_{j=1}^{4} \delta\left(\boldsymbol{e}_{2}\right) \delta\left(\boldsymbol{e}_{j}\right) \omega_{2 j} \wedge \omega_{j}=\sum_{j=3}^{4} \delta\left(\boldsymbol{e}_{j}\right) \omega_{2 j} \wedge \omega_{j}=\omega_{23} \wedge \omega_{3}+\omega_{24} \wedge \omega_{4} .
\end{array}\right.
$$

By Cartan's lemma, we can then write

$$
\left\{\begin{array}{l}
\omega_{13}=a \omega_{3}+b \omega_{4}, \omega_{14}=b \omega_{3}+c \omega_{4}, \\
\omega_{23}=e \omega_{3}+f \omega_{4}, \omega_{24}=f \omega_{3}+g \omega_{4}
\end{array}\right.
$$


for appropriate functions $a, b, c, e, f$ and $g$. We define that $\left\langle d^{2} \boldsymbol{X}, \boldsymbol{e}_{i}\right\rangle=-\left\langle d \boldsymbol{X}, d \boldsymbol{e}_{i}\right\rangle, i=$ 1,2 , then we have a vector-valued quadratic form:

$$
-\left\langle d^{2} \boldsymbol{X}, \boldsymbol{e}_{1}\right\rangle \boldsymbol{e}_{1}+\left\langle d^{2} \boldsymbol{X}, e_{2}\right\rangle \boldsymbol{e}_{2}=\left(a \omega_{3}^{2}+2 b \omega_{3} \omega_{4}+c \omega_{4}^{2}\right) \boldsymbol{e}_{1}-\left(e \omega_{3}^{2}+2 f \omega_{3} \omega_{4}+g \omega_{4}^{2}\right) \boldsymbol{e}_{2},
$$

which is called the second fundamental form of the spacelike surface. It follows from $\left(^{*}\right)$ that

$$
d\left(\begin{array}{l}
\boldsymbol{e}_{1} \\
\boldsymbol{e}_{2} \\
\boldsymbol{e}_{3} \\
\boldsymbol{e}_{4}
\end{array}\right)=\left(\begin{array}{cccc}
0 & \omega_{12} & \omega_{13} & \omega_{14} \\
\omega_{12} & 0 & \omega_{23} & \omega_{24} \\
\omega_{13} & -\omega_{23} & 0 & \omega_{34} \\
\omega_{14} & -\omega_{24} & -\omega_{34} & 0
\end{array}\right)\left(\begin{array}{l}
\boldsymbol{e}_{1} \\
\boldsymbol{e}_{2} \\
\boldsymbol{e}_{3} \\
\boldsymbol{e}_{4}
\end{array}\right)
$$

from which we also get the following equations:

$$
d\left(\begin{array}{c}
\boldsymbol{e}_{1}-\boldsymbol{e}_{2} \\
\boldsymbol{e}_{1}+\boldsymbol{e}_{2} \\
\boldsymbol{e}_{3} \\
\boldsymbol{e}_{4}
\end{array}\right)=\left(\begin{array}{cccc}
0 & -\omega_{12} & \omega_{13}-\omega_{23} & \omega_{14}-\omega_{24} \\
\omega_{12} & 0 & \omega_{13}+\omega_{23} & \omega_{14}+\omega_{24} \\
\frac{\omega_{13}-\omega_{23}}{\frac{\omega_{14}-\omega_{24}}{2}} & \frac{\omega_{13}+\omega_{23}}{2} & 0 & \omega_{14}+\omega_{24} \\
2 & -\omega_{34} & 0
\end{array}\right)\left(\begin{array}{c}
\boldsymbol{e}_{1}+\boldsymbol{e}_{2} \\
\boldsymbol{e}_{1}-\boldsymbol{e}_{2} \\
\boldsymbol{e}_{3} \\
\boldsymbol{e}_{4}
\end{array}\right)
$$

On the other hand, we define

$$
L C_{p}=\left\{\boldsymbol{x} \in \mathbb{R}_{1}^{4} \mid-\left(x_{1}-p_{1}\right)^{2}+\sum_{i=2}^{4}\left(x_{i}-p_{i}\right)^{2}=0\right\}
$$

and

$$
S_{+}^{2}=\left\{\boldsymbol{x}=\left(x_{1}, x_{2}, x_{3}, x_{4}\right) \in L C_{0} \mid x_{1}=1\right\},
$$

where $p=\left(p_{1}, p_{2}, p_{3}, p_{4}\right) \in \mathbb{R}_{1}^{4}$. We call $S_{+}^{2}$ the (future) spacelike unit sphere and $L C_{p}^{*}=$ $L C_{p} \backslash\{p\}$ the lightcone with deleted vertex at $p$. We also define

$$
L C_{+}^{*}=\left\{\boldsymbol{x}=\left(x_{1}, x_{2}, x_{3}, x_{4}\right) \in L C_{0}^{*} \mid x_{1}>0\right\}
$$

and call it a future lightcone at the origin. For any lightlike vector $\boldsymbol{x}=\left(x_{1}, x_{2}, x_{3}, x_{4}\right)$, we have

$$
\widetilde{\boldsymbol{x}}=\left(1, \frac{x_{2}}{x_{1}}, \frac{x_{3}}{x_{1}}, \frac{x_{4}}{x_{1}},\right) \in S_{+}^{2} .
$$

Let $\boldsymbol{e}_{1}=\left(a_{1}, a_{2}, a_{3}, a_{4}\right)$ and $\boldsymbol{e}_{2}=\left(b_{1}, b_{2}, b_{3}, b_{4}\right)$. Clearly, we have

$$
d\left(\boldsymbol{e}_{1} \pm \boldsymbol{e}_{2}\right)=d\left(a_{1} \pm b_{1}\right)\left(\widetilde{\boldsymbol{e}_{1} \pm \boldsymbol{e}_{2}}\right)+\left(a_{1} \pm b_{1}\right) d\left(\widetilde{\boldsymbol{e}_{1} \pm \boldsymbol{e}_{2}}\right) .
$$


Finally, we get the following fundamental formula:

$$
d\left(\begin{array}{c}
\widetilde{\boldsymbol{e}_{1}-\boldsymbol{e}_{2}} \\
\widetilde{\boldsymbol{e}_{1}+\boldsymbol{e}_{2}} \\
\boldsymbol{e}_{3} \\
\boldsymbol{e}_{4}
\end{array}\right)=\left(\begin{array}{cccc}
0 & -\omega_{12}-\frac{d\left(a_{1}-b_{1}\right)}{a_{1}-b_{1}} & \frac{\omega_{13}-\omega_{23}}{a_{1}-b_{1}} & \frac{\omega_{14}-\omega_{24}}{a_{1}-b_{1}} \\
\omega_{12}-\frac{d\left(a_{1}+b_{1}\right)}{a_{1}+b_{1}} & 0 & \frac{\omega_{13}+\omega_{23}}{a_{1}+b_{1}} & \frac{\omega_{14}+\omega_{24}}{a_{1}+b_{1}} \\
\frac{\omega_{13}-\omega_{23}}{\omega_{14} \frac{2}{2} \omega_{24}} & \frac{\omega_{13}+\omega_{23}}{2} & 0 & \omega_{34} \\
\frac{\omega_{14}+\omega_{24}}{2} & -\omega_{34} & 0
\end{array}\right)\left(\begin{array}{c}
\widetilde{\boldsymbol{e}_{1}+\boldsymbol{e}_{2}} \\
\widetilde{\boldsymbol{e}_{1}-\boldsymbol{e}_{2}} \\
\boldsymbol{e}_{3} \\
\boldsymbol{e}_{4}
\end{array}\right) .
$$

For a given normal vector $\boldsymbol{v}=\xi \boldsymbol{e}_{1}+\eta \boldsymbol{e}_{2} \in N_{p} M$, we have $d \boldsymbol{v}=d \xi \boldsymbol{e}_{1}+\xi d \boldsymbol{e}_{1}+d \eta \boldsymbol{e}_{2}+\eta d \boldsymbol{e}_{2}$ and hence

$$
\begin{aligned}
\left\langle d \boldsymbol{v}, \boldsymbol{e}_{3}\right\rangle \wedge\left\langle d \boldsymbol{v}, \boldsymbol{e}_{4}\right\rangle & =\left[(a \xi+e \eta)(c \xi+g \eta)-(b \xi+f \eta)^{2}\right] \omega_{3} \wedge \omega_{4} \\
& =\left[\left(a c-b^{2}\right) \xi^{2}+(e c+a g-2 b f) \xi \eta+\left(e g-f^{2}\right) \eta^{2}\right] \omega_{3} \wedge \omega_{4} .
\end{aligned}
$$

We define a function $\mathcal{K}_{l}$ as follows:

$$
\mathcal{K}_{l}(\boldsymbol{v})(p)=\mathcal{K}_{l}(\xi, \eta)(p)=\left(a c-b^{2}\right) \xi^{2}+(e c+a g-2 b f) \xi \eta+\left(e g-f^{2}\right) \eta^{2} .
$$

We also define the mean curvature vector $\mathfrak{H}$ by

$$
\mathfrak{H}(p)=\frac{1}{2}(a+c) \boldsymbol{e}_{1}-\frac{1}{2}(e+g) \boldsymbol{e}_{2}
$$

and

$$
H_{l}(\boldsymbol{v})(p)=H_{l}(\xi, \eta)(p)=\langle\mathfrak{H}(p), \boldsymbol{v}\rangle=\frac{1}{2}(a+c) \xi+\frac{1}{2}(e+g) \eta .
$$

We now consider a symmetric matrix

$$
A^{ \pm}=\left(\begin{array}{ll}
a \pm e & b \pm f \\
b \pm f & c \pm g
\end{array}\right) .
$$

Let $\kappa_{i}^{ \pm}(p), i=1,2$ be the eigenvalues of $A^{ \pm}$which we call principal lightcone curvatures of $M$ at $p$. By definition, we have

$$
\kappa_{1}^{ \pm}(p) \kappa_{2}^{ \pm}(p)=\operatorname{det} A=\left(a c-b^{2}\right) \pm(c e+a g-2 b f)+\left(e g-f^{2}\right)=K_{l}(1, \pm 1)(p)
$$

and

$$
2 H_{l}(1, \pm 1)(p)= \pm e \pm g+a+c=\kappa_{1}^{ \pm}(p)+\kappa_{2}^{ \pm}(p) .
$$

We say that $p \in M$ is an umbilic point if $\kappa_{1}^{ \pm}(p)=\kappa_{2}^{ \pm}(p)$. An umbilic point is flat if $K_{l}(1, \pm 1)(p)=0$. On the other hand, we define a pair of hypersurfaces

$$
L H_{M}^{ \pm}: M \times \mathbb{R} \longrightarrow \mathbb{R}_{1}^{4}
$$

by

$$
L H_{M}^{ \pm}(p, u)=L H_{M}^{ \pm}(x, y, u)=\boldsymbol{X}(x, y)+u\left(\widetilde{\boldsymbol{e}_{1} \pm \boldsymbol{e}_{2}}\right)(x, y),
$$

where $p=\boldsymbol{X}(x, y)$. We call $L H_{M}^{ \pm}$the lightlike hypersurface along $M$.

In general, a hypersurface $H \subset \mathbb{R}_{1}^{4}$ is called a lightlike hypersurface if it is tangent to a lightcone at any point. It is known that any lightlike hypersurface is given by the construction above at least locally (cf. [10] and §6). 


\section{Lorentzian distance-squared functions on spacelike surfaces}

In this section we introduce the notion of Lorentzian distance-squared functions on spacelike surfaces, which is useful for the study of singularities of lightlike hypersurfaces.

First we define a family of functions $G: M \times \mathbb{R}_{1}^{4} \longrightarrow \mathbb{R}$ on a spacelike surface $M=$ $\boldsymbol{X}(U)$ by

$$
G(p, \boldsymbol{\lambda})=G(x, y, \boldsymbol{\lambda})=\langle\boldsymbol{X}(x, y)-\boldsymbol{\lambda}, \boldsymbol{X}(x, y)-\boldsymbol{\lambda}\rangle,
$$

where $p=\boldsymbol{X}(x, y)$. We call $G$ the Lorentzian distance-squared function on the spacelike surface $M$. For any fixed $\boldsymbol{\lambda}_{0} \in \mathbb{R}_{1}^{4}$, we write $g(p)=G_{\lambda_{0}}(p)=G\left(p, \boldsymbol{\lambda}_{0}\right)$ and have the following proposition.

Proposition 3.1 Let $M$ be a spacelike surface and $G: M \times \mathbb{R}_{1}^{4} \rightarrow \mathbb{R}$ the Lorentzian distance-squared function on $M$. Suppose that $p_{0} \neq \boldsymbol{\lambda}_{0}$. Then we have the following:

(1) $g\left(p_{0}\right)=\partial g / \partial x\left(p_{0}\right)=\partial g / \partial y\left(p_{0}\right)=0$ if and only if $p_{0}-\boldsymbol{\lambda}_{0}=\mu\left(\boldsymbol{e}_{1} \pm \boldsymbol{e}_{2}\right)\left(p_{0}\right)$ for some $\mu \in \mathbb{R} \backslash\{0\}$.

(2) $g\left(p_{0}\right)=\partial g / \partial x\left(p_{0}\right)=\partial g / \partial y\left(p_{0}\right)=\operatorname{det} \mathcal{H}(g)\left(p_{0}\right)=0\left(\operatorname{det} \mathcal{H}(g)\left(p_{0}\right)\right.$ is the determinant of the Hessian matrix) if and only if

$$
p_{0}-\boldsymbol{\lambda}_{0}=\mu\left(\widetilde{\boldsymbol{e}_{1} \pm \boldsymbol{e}_{2}}\right)\left(p_{0}\right)
$$

for some $\mu \in \mathbb{R} \backslash\{0\}$ which is the inverse of a non-zero principal curvature $\kappa_{i}^{\mp}\left(p_{0}\right), i=1,2$.

Proof. (1) The condition $g(p)=\left\langle\boldsymbol{X}(x, y)-\boldsymbol{\lambda}_{0}, \boldsymbol{X}(x, y)-\boldsymbol{\lambda}_{0}\right\rangle=0$ means that $\boldsymbol{X}(x, y)-$ $\boldsymbol{\lambda}_{0} \in L C_{0}$. We can observe that $d g(p)=\left\langle d \boldsymbol{X}(x, y), \boldsymbol{X}(x, y)-\boldsymbol{\lambda}_{0}\right\rangle=0$ if and only if $\boldsymbol{X}(x, y)-\boldsymbol{\lambda}_{0} \in N_{p} M$. Hence $g\left(p_{0}\right)=d g\left(p_{0}\right)=0$ if and only if $p_{0}-\boldsymbol{\lambda}_{0} \in N_{p} M \cap L C_{0}$. This is equivalent to the condition that $p_{0}-\boldsymbol{\lambda}_{0}=\mu\left(\widetilde{\boldsymbol{e}_{1} \pm \boldsymbol{e}_{2}}\right)\left(p_{0}\right)$ for some $\mu \in \mathbb{R} \backslash\{0\}$.

(2) By a Lorentzian motion, we may assume that $p_{0}$ is the origin of $\mathbb{R}_{1}^{4}$. We can choose local coordinates such that $\boldsymbol{X}$ is given by the Monge form

$$
\boldsymbol{X}(x, y)=\left(f_{1}(x, y), f_{2}(x, y), x, y\right)
$$

with $f_{1_{x}}(0,0)=f_{1_{y}}(0,0)=f_{2_{x}}(0,0)=f_{2_{y}}(0,0)=0$, so that we have $\boldsymbol{e}_{1}\left(p_{0}\right)=(1,0,0,0)$ and $\boldsymbol{e}_{2}\left(p_{0}\right)=(0,1,0,0)$. In this case we have

$$
\begin{aligned}
& f_{1_{x x}}(0,0)=-a\left(p_{0}\right), f_{1_{x y}}(0,0)=-b\left(p_{0}\right), f_{1_{y y}}(0,0)=-c\left(p_{0}\right), \\
& f_{2_{x x}}(0,0)=e\left(p_{0}\right), f_{2_{x y}}(0,0)=f\left(p_{0}\right), f_{2_{y y}}(0,0)=g\left(p_{0}\right) .
\end{aligned}
$$

Under the condition (1), we have the following calculations:

$$
\begin{aligned}
\frac{\partial^{2} g}{\partial x^{2}} & =g_{x x}=2\left(\left\langle\boldsymbol{X}_{x x}, \boldsymbol{X}-\boldsymbol{\lambda}_{0}\right\rangle+\left\langle\boldsymbol{X}_{x}, \boldsymbol{X}_{x}\right\rangle\right) \\
& =2\left(\left\langle\left(f_{1_{x x}}, f_{2_{x x}}, 0,0\right), \mu\left(\widetilde{\boldsymbol{e}_{1} \pm \boldsymbol{e}_{2}}\right)\left(p_{0}\right)\right\rangle+2\left\langle\left(f_{1_{x}}, f_{2_{x}}, 1,0\right),\left(f_{1_{x}}, f_{2_{x}}, 1,0\right)\right\rangle\right),
\end{aligned}
$$




$$
\begin{aligned}
\frac{\partial^{2} g}{\partial x \partial y} & =g_{x y}=2\left(\left\langle\boldsymbol{X}_{x y}, \boldsymbol{X}-\boldsymbol{\lambda}_{0}\right\rangle+\left\langle\boldsymbol{X}_{x}, \boldsymbol{X}_{y}\right\rangle\right) \\
& =2\left\langle\left(f_{1_{x y}}, f_{2_{x y}}, 0,0\right), \mu\left(\widetilde{\boldsymbol{e}_{1} \pm \boldsymbol{e}_{2}}\right)\left(p_{0}\right)\right\rangle+2\left\langle\left(f_{1_{x}}, f_{2_{x}}, 1,0\right),\left(f_{1_{y}}, f_{2_{y}}, 0,1\right)\right\rangle \\
\frac{\partial^{2} g}{\partial y^{2}} & =g_{y y}=2\left(\left\langle\boldsymbol{X}_{y y}, \boldsymbol{X}-\boldsymbol{\lambda}_{0}\right\rangle+\left\langle\boldsymbol{X}_{y}, \boldsymbol{X}_{y}\right\rangle\right) \\
& =2\left\langle\left(f_{1_{y y}}, f_{2_{y y}}, 0,0\right), \mu\left(\widetilde{\boldsymbol{e}_{1} \pm \boldsymbol{e}_{2}}\right)\left(p_{0}\right)\right\rangle+2\left\langle\left(f_{1_{y}}, f_{2_{y}}, 0,1\right),\left(f_{1_{y}}, f_{2_{y}}, 0,1\right)\right\rangle .
\end{aligned}
$$

It follows that

$$
\begin{aligned}
& g_{x x}(0,0)=-2 \mu a\left(p_{0}\right) \pm 2 \mu e\left(p_{0}\right)+2, \\
& g_{x y}(0,0)=-2 \mu b\left(p_{0}\right) \pm 2 \mu f\left(p_{0}\right), \\
& g_{y y}(0,0)=-2 \mu c\left(p_{0}\right) \pm 2 \mu g\left(p_{0}\right)+2 .
\end{aligned}
$$

Therefore,

$$
\operatorname{det} \mathcal{H}\left(g_{\lambda}\right)\left(p_{0}\right)=\left|\begin{array}{cc}
-\mu a \pm \mu e+1 & -\mu b \pm \mu f \\
-\mu b \pm \mu f & -\mu c \pm \mu g+1
\end{array}\right|\left(p_{0}\right)=0
$$

if and only if

$$
\left(a c+e g \mp a g \mp c e-b^{2}-f^{2} \pm 2 b f\right) \mu^{2}+( \pm e \pm g-a-c) \mu+1=0,
$$

which is equivalent to

$$
K_{l}(1, \mp 1) \mu^{2}-2 H_{l}(1, \mp 1) \mu+1=0 .
$$

This means that $\mu \neq 0$ and $1 / \mu$ is one of the lightcone principal curvatures $\kappa_{i}^{\mp}\left(p_{0}\right)$.

Thus Proposition 3.1 means that the discriminant set of the Lorentzian distancesquared function $G$ is given by

$$
\mathcal{D}_{G}=\left\{\boldsymbol{\lambda} \mid \boldsymbol{\lambda}=\boldsymbol{X}(p)+u\left(\widetilde{\boldsymbol{e}_{1} \pm \boldsymbol{e}_{2}}\right)(p), p \in M, u \in \mathbb{R}\right\},
$$

which is the image of the lightlike hypersurface along $M$. Therefore a singular point of the lightlike hypersurface is a point $\boldsymbol{\lambda}_{0}=\boldsymbol{X}\left(p_{0}\right)+u_{0}\left(\boldsymbol{e}_{1} \pm \boldsymbol{e}_{2}\right)\left(p_{0}\right)$ at which $u_{0}=-1 / \kappa_{i}^{\mp}\left(p_{0}\right)$, $i=1,2$.

We now explain the reason why such a correspondence exists from the point of view of contact geometry. Let $\pi: P T^{*}\left(\mathbb{R}_{1}^{4}\right) \longrightarrow \mathbb{R}_{1}^{4}$ be the projective cotangent bundle with its canonical contact structure. We next review the geometric properties of this bundle. Consider the tangent bundle $\tau: T P T^{*}\left(\mathbb{R}_{1}^{4}\right) \rightarrow P T^{*}\left(\mathbb{R}_{1}^{4}\right)$ and the differential map $d \pi$ : $T P T^{*}\left(\mathbb{R}_{1}^{4}\right) \rightarrow T \mathbb{R}_{1}^{4}$ of $\pi$. For any $X \in T P T^{*}\left(\mathbb{R}_{1}^{4}\right)$, there exists an element $\alpha \in T^{*}\left(\mathbb{R}_{1}^{4}\right.$ such that $\tau(X)=[\alpha]$. For an element $V \in T_{x}\left(\mathbb{R}_{1}^{4}\right)$, the property $\alpha(V)=0$ does not depend 
on the choice of representative of the class $[\alpha]$. Thus we can define the canonical contact structure on $P T^{*}\left(\mathbb{R}_{1}^{4}\right)$ by

$$
K=\left\{X \in T P T^{*}\left(\mathbb{R}_{1}^{4}\right) \mid \tau(X)(d \pi(X))=0\right\}
$$

Via the coordinates $\left(v_{1}, v_{2}, v_{3}, v_{4}\right)$, we have the trivialization $P T^{*}\left(\mathbb{R}_{1}^{4}\right) \cong \mathbb{R}_{1}^{4} \times P^{3}(\mathbb{R})^{*}$, and call

$$
\left(\left(v_{1}, v_{2}, v_{3}, v_{4}\right),\left[\xi_{1}: \xi_{2}: \xi_{3}: \xi_{4}\right]\right)
$$

homogeneous coordinates of $P T^{*}\left(\mathbb{R}_{1}^{4}\right)$, where $\left[\xi_{1}: \xi_{2}: \xi_{3}: \xi_{4}\right]$ are the homogeneous coordinates of the dual projective space $P^{3}(\mathbb{R})^{*}$.

It is easy to show that $X \in K_{(x,[\xi])}$ if and only if $\sum_{i=1}^{4} \mu_{i} \xi_{i}=0$, where $d \tilde{\pi}(X)=$ $\sum_{i=1}^{4} \mu_{i} \partial / \partial v_{i}$. An immersion $i: L \rightarrow P T^{*}\left(\mathbb{R}_{1}^{4}\right)$ is said to be a Legendrian immersion if $\operatorname{dim} L=3$ and $d i_{q}\left(T_{q} L\right) \subset K_{i(q)}$ for any $q \in L$. The map $\pi \circ i$ is also called the Legendrian map and the set $W(i)=\operatorname{image} \pi \circ i$, the wave front of $i$. Moreover, $i$ (or, the image of $i$ ) is called the Legendrian lift of $W(i)$. In the appendix, we give a quick survey of the theory of Legendrian singularities. For additional definitions and basic results on generating families, we refer to ([1], Chapter 21). By the preceding arguments, the lightlike hypersurface $L H_{M}^{ \pm}$is the discriminant set of the Lorentzian distance-squared function $G$. We have the following proposition (See the appendix for the definition of a Morse family).

Proposition 3.2 Let $G$ be the Lorentzian distance-squared function on $M$. For any point $((x, y), \boldsymbol{\lambda}) \in G^{-1}(0), G$ is a Morse family around $((x, y), \boldsymbol{\lambda})$.

Proof. Denote

$$
\boldsymbol{X}(x, y)=\left(X_{1}(x, y), X_{2}(x, y), X_{3}(x, y), X_{4}(x, y)\right) \text { and } \boldsymbol{\lambda}=\left(\lambda_{1}, \lambda_{2}, \lambda_{3}, \lambda_{4}\right)
$$

By definition, we have

$$
G(x, y, \boldsymbol{\lambda})=-\left(X_{1}(x, y)-\lambda_{1}\right)^{2}+\left(X_{2}(x, y)-\lambda_{2}\right)^{2}+\left(X_{3}(x, y)-\lambda_{3}\right)^{2}+\left(X_{4}(x, y)-\lambda_{4}\right)^{2}
$$

We now prove that the mapping

$$
\Delta^{*} G=\left(G, \frac{\partial G}{\partial x}, \frac{\partial G}{\partial y}\right)
$$

is non-singular at $((x, y), \boldsymbol{\lambda}) \in G^{-1}(0)$. Indeed, the Jacobian matrix of $\Delta^{*} G$ is given by

$$
\left(\begin{array}{ccccc} 
& 2\left(X_{1}-\lambda_{1}\right) & -2\left(X_{2}-\lambda_{2}\right) & -2\left(X_{3}-\lambda_{3}\right) & -2\left(X_{4}-\lambda_{4}\right) \\
\mathrm{A} & 2 X_{1 x} & -2 X_{2 x} & -2 X_{3 x} & -2 X_{4 x} \\
2 X_{1 y} & -2 X_{2 y} & -2 X_{3 y} & -2 X_{4 y}
\end{array}\right)
$$


where

$$
\boldsymbol{A}=\left(\begin{array}{cc}
2\left\langle\boldsymbol{X}-\boldsymbol{\lambda}, \boldsymbol{X}_{x}\right\rangle & 2\left\langle\boldsymbol{X}-\boldsymbol{\lambda}, \boldsymbol{X}_{y}\right\rangle \\
2\left(\left\langle\boldsymbol{X}_{x}, \boldsymbol{X}_{x}\right\rangle+\left\langle\boldsymbol{X}-\boldsymbol{\lambda}, \boldsymbol{X}_{x x}\right\rangle\right) & 2\left(\left\langle\boldsymbol{X}_{x}, \boldsymbol{X}_{y}\right\rangle+\left\langle\boldsymbol{X}-\boldsymbol{\lambda}, \boldsymbol{X}_{x y}\right\rangle\right) \\
2\left(\left\langle\boldsymbol{X}_{y}, \boldsymbol{X}_{x}\right\rangle+\left\langle\boldsymbol{X}-\boldsymbol{\lambda}, \boldsymbol{X}_{y x}\right\rangle\right) & 2\left(\left\langle\boldsymbol{X}_{y}, \boldsymbol{X}_{y}\right\rangle+\left\langle\boldsymbol{X}-\boldsymbol{\lambda}, \boldsymbol{X}_{y y}\right\rangle\right)
\end{array}\right) .
$$

Since $\boldsymbol{X}$ is an immersion, the rank of the matrix

$$
\left(\begin{array}{llll}
2 X_{1 x} & -2 X_{2 x} & -2 X_{3 x} & -2 X_{4 x} \\
2 X_{1 y} & -2 X_{2 y} & -2 X_{3 y} & -2 X_{4 y}
\end{array}\right)
$$

is equal to two. Moreover, $\boldsymbol{X}-\boldsymbol{\lambda}$ is lightlike, so that it is linearly independent of tangent vectors $\boldsymbol{X}_{x}, \boldsymbol{X}_{y}$. This means that the rank of the matrix

$$
\left(\begin{array}{cccc}
2\left(X_{1}-\lambda_{1}\right) & -2\left(X_{2}-\lambda_{2}\right) & -2\left(X_{3}-\lambda_{3}\right) & -2\left(X_{4}-\lambda_{4}\right) \\
2 X_{1 x} & -2 X_{2 x} & -2 X_{3 x} & -2 X_{4 x} \\
2 X_{1 y} & -2 X_{2 y} & -2 X_{3 y} & -2 X_{4 y}
\end{array}\right)
$$

is equal to three. Therefore the Jacobi matrix of $\Delta^{*} G$ is non-singular at $((x, y), \boldsymbol{\lambda}) \in$ $G^{-1}(0)$.

Since $G$ is a Morse family, we can define a Legendrian immersion

$$
L_{G}^{ \pm}: \Sigma_{*}(G) \longrightarrow P T^{*}\left(\mathbb{R}_{1}^{4}\right)
$$

by

$$
L_{G}^{ \pm}(x, y, \boldsymbol{\lambda})=\left(\boldsymbol{\lambda},\left[\left(X_{1}(x, y)-\lambda_{1}\right):\left(\lambda_{2}-X_{2}(x, y)\right):\left(\lambda_{3}-X_{3}(x, y)\right):\left(\lambda_{4}-X_{4}(x, y)\right)\right]\right),
$$

where

$$
\Sigma_{*}(G)=\left(\Delta^{*} G\right)^{-1}(0)=\left\{(x, y, \boldsymbol{\lambda}) \mid \boldsymbol{\lambda}=L H_{M}^{ \pm}(x, y, u) \text { for some } u \in \mathbb{R}\right\} .
$$

We observe that $G$ is a generating family of the Legendrian immersion $L_{G}^{ \pm}$whose wave front is $L H_{M}^{ \pm}$(cf. the appendix). Therefore we might say that the Lorentzian distance-squared function $G$ on $M$ gives a Minkowski-canonical generating family for the Legendrian lift of $L H_{M}^{ \pm}$.

\section{Contact with lightcones}

In this section we describe Montaldi's characterization of submanifolds contact in terms of $\mathcal{K}$-equivalence. It is then adapted to lightlike hypersurfaces and their indicatrices. We begin with the following basic observations. 
Proposition 4.1 Let $\boldsymbol{\lambda}_{0} \in \mathbb{R}_{1}^{4}$ and $M$ a spacelike surface without umbilic points satisfying $K_{l}(1, \mp 1) \neq 0$. Then $M \subset L C_{\lambda_{0}}$ if and only if $\boldsymbol{\lambda}_{0}$ is an isolated singular value of the lightlike hypersurface $L H_{M}^{ \pm}$and $L H_{M}^{ \pm}(U \times \mathbb{R}) \subset L C_{\lambda_{0}}$.

Proof. By definition, $M \subset L C_{\lambda_{0}}$ if and only if $g_{\lambda_{0}}(x, y) \equiv 0$ for any $(x, y) \in U$, where $g_{\lambda_{0}}(x, y)=G\left(x, y, \boldsymbol{\lambda}_{0}\right)$ is the Lorentzian distance-squared function on $M$. It follows from Proposition 3.1 that there exists a smooth function $\mu: U \longrightarrow \mathbb{R}$ such that

$$
\boldsymbol{X}(x, y)=\boldsymbol{\lambda}_{0}+\mu(x, y)\left(\widetilde{\boldsymbol{e}_{1} \pm \boldsymbol{e}_{2}}\right)(x, y)
$$

Therefore we have

$$
L H_{M}^{ \pm}(x, y, u)=\boldsymbol{\lambda}_{0}+(u+\mu(x, y))\left(\widetilde{\boldsymbol{e}_{1} \pm \boldsymbol{e}_{2}}\right)(x, y) .
$$

Hence we have $L H_{M}^{ \pm}(U \times \mathbb{R}) \subset L C_{\lambda_{0}}$. Moreover, it follows that

$$
\begin{aligned}
& \frac{\partial L H_{M}^{ \pm}}{\partial u}=\left(\widetilde{\boldsymbol{e}_{1} \pm \boldsymbol{e}_{2}}\right)(x, y) \\
& \frac{\partial L H_{M}^{ \pm}}{\partial x}=\mu_{x}(x, y)\left(\widetilde{\boldsymbol{e}_{1} \pm \boldsymbol{e}_{2}}\right)(x, y)+(u+\mu(x, y))\left(\widetilde{\boldsymbol{e}_{1} \pm \boldsymbol{e}_{2}}\right)_{x}(x, y), \\
& \frac{\partial L H_{M}^{ \pm}}{\partial y}=\mu_{y}(x, y)\left(\widetilde{\boldsymbol{e}_{1} \pm \boldsymbol{e}_{2}}\right)(x, y)+(u+\mu(x, y))\left(\widetilde{\boldsymbol{e}_{1} \pm \boldsymbol{e}_{2}}\right)_{y}(x, y),
\end{aligned}
$$

from which we obtain

$$
\left(\frac{\partial L H_{M}^{ \pm}}{\partial u} \wedge \frac{\partial L H_{M}^{ \pm}}{\partial x} \wedge \frac{\partial L H_{M}^{ \pm}}{\partial y}\right)=(u+\mu(x, y))^{2}\left(\widetilde{\boldsymbol{e}_{1} \pm \boldsymbol{e}_{2}}\right) \wedge\left(\widetilde{\boldsymbol{e}_{1} \pm \boldsymbol{e}_{2}}\right)_{x} \wedge\left(\widetilde{\boldsymbol{e}_{1} \pm \boldsymbol{e}_{2}}\right)_{y} .
$$

By the assumption, we have

$$
\boldsymbol{X}-\boldsymbol{\lambda}_{0}=\mu(x, y)\left(\widetilde{\boldsymbol{e}_{1} \pm \boldsymbol{e}_{2}}\right)(x, y)
$$

Since $\boldsymbol{X}-\boldsymbol{\lambda}_{0}$ is lightlike and $\boldsymbol{X}_{x}, \boldsymbol{X}_{y}$ are spacelike, $\boldsymbol{X}-\boldsymbol{\lambda}_{0}, \boldsymbol{X}_{x}, \boldsymbol{X}_{y}$ are linearly independent. Therefore we have

$$
\mathbf{0} \neq\left(\boldsymbol{X}-\boldsymbol{\lambda}_{0}\right) \wedge \boldsymbol{X}_{x} \wedge \boldsymbol{X}_{y}=\mu(x, y)^{3}\left(\widetilde{\boldsymbol{e}_{1} \pm \boldsymbol{e}_{2}}\right) \wedge\left(\widetilde{\boldsymbol{e}_{1} \pm \boldsymbol{e}_{2}}\right)_{x} \wedge\left(\widetilde{\boldsymbol{e}_{1} \pm \boldsymbol{e}_{2}}\right)_{y},
$$

so that

$$
\left(\frac{\partial L H_{M}^{ \pm}}{\partial u} \wedge \frac{\partial L H_{M}^{ \pm}}{\partial x} \wedge \frac{\partial L H_{M}^{ \pm}}{\partial y}\right)=0
$$

if and only if $u+\mu(x, y)=0$ under the assumption that $K_{l}(1, \mp 1) \neq 0$. This means that $\boldsymbol{\lambda}_{0}$ is an isolated singularity of $L H_{M}^{ \pm}$. The converse assertion is trivial.

Motivated by the proposition above, we now consider the contact of spacelike surfaces with lightcones in view of Montaldi's theorem [15]. Let $X_{i}$ and $Y_{i}, i=1,2$, be submanifolds 
of $\mathbb{R}^{n}$ with $\operatorname{dim} X_{1}=\operatorname{dim} X_{2}$ and $\operatorname{dim} Y_{1}=\operatorname{dim} Y_{2}$. We say that the contact of $X_{1}$ and $Y_{1}$ at $y_{1}$ is same type as the contact of $X_{2}$ and $Y_{2}$ at $y_{2}$ if there is a diffeomorphism germ $\Phi:\left(\mathbb{R}^{n}, y_{1}\right) \longrightarrow\left(\mathbb{R}^{n}, y_{2}\right)$ such that $\Phi\left(X_{1}\right)=X_{2}$ and $\Phi\left(Y_{1}\right)=Y_{2}$. In this case we write $K\left(X_{1}, Y_{1} ; y_{1}\right)=K\left(X_{2}, Y_{2} ; y_{2}\right)$. Since this definition of contact is local, we can replace $\mathbb{R}^{n}$ by arbitrary $n$-manifold. Montaldi gives in [15] the following characterization of contact by using $\mathcal{K}$-equivalence.

Theorem 4.2 Let $X_{i}$ and $Y_{i}, i=1,2$, be submanifolds of $\mathbb{R}^{n}$ with $\operatorname{dim} X_{1}=\operatorname{dim} X_{2}$ and $\operatorname{dim} Y_{1}=\operatorname{dim} Y_{2}$. Let $g_{i}:\left(X_{i}, x_{i}\right) \longrightarrow\left(\mathbb{R}^{n}, y_{i}\right)$ be immersion germs and $f_{i}:\left(\mathbb{R}^{n}, y_{i}\right) \longrightarrow$ $\left(\mathbb{R}^{p}, 0\right)$ be submersion germs with $\left(Y_{i}, y_{i}\right)=\left(f_{i}^{-1}(0), y_{i}\right)$. Then

$$
K\left(X_{1}, Y_{1} ; y_{1}\right)=K\left(X_{2}, Y_{2} ; y_{2}\right)
$$

if and only if $f_{1} \circ g_{1}$ and $f_{2} \circ g_{2}$ are $\mathcal{K}$-equivalent.

Turning to lightlike hypersurfaces, we now consider the function $\mathcal{G}: \mathbb{R}_{1}^{4} \times \mathbb{R}_{1}^{4} \longrightarrow \mathbb{R}$ defined by $\mathcal{G}(\boldsymbol{x}, \boldsymbol{\lambda})=\langle\boldsymbol{x}-\boldsymbol{\lambda}, \boldsymbol{x}-\boldsymbol{\lambda}\rangle$. Given $\boldsymbol{\lambda}_{0} \in \mathbb{R}_{1}^{4}$, we denote $\mathfrak{g}_{\lambda_{0}}(\boldsymbol{x})=\mathcal{G}\left(\boldsymbol{x}, \boldsymbol{\lambda}_{0}\right)$, so that we have $\mathfrak{g}_{\lambda_{0}}^{-1}(0)=L C_{\lambda_{0}}$. For any $\left(x_{0}, y_{0}\right) \in U$, we take the point $\boldsymbol{\lambda}_{0}^{ \pm}=\boldsymbol{X}\left(x_{0}, y_{0}\right)+$ $u_{0}\left(\widetilde{\boldsymbol{e}_{1} \pm \boldsymbol{e}_{2}}\right)\left(x_{0}, y_{0}\right)$ and have

$$
\left.\mathfrak{g}_{\lambda_{0}^{ \pm}} \circ \boldsymbol{X}\left(x_{0}, y_{0}\right)\right)=\mathcal{G} \circ\left(\boldsymbol{X} \times i d_{\mathbb{R}_{1}^{4}}\right)\left(\left(x_{0}, y_{0}\right), \boldsymbol{\lambda}_{0}^{ \pm}\right)=G\left(x_{0}, y_{0}, \boldsymbol{\lambda}_{0}^{ \pm}\right)=0,
$$

where $u_{0}=-1 / \kappa_{i}^{\mp}\left(x_{0}, y_{0}\right), i=1,2$. We also have relations

$$
\frac{\partial \mathfrak{g}_{\lambda_{0}^{ \pm}} \circ \boldsymbol{X}}{\partial x}\left(p_{0}\right)=\frac{\partial G}{\partial x}\left(\left(p_{0}\right), \boldsymbol{\lambda}_{0}^{ \pm}\right)=0, \frac{\partial \mathfrak{g}_{\lambda_{0}^{ \pm}} \circ \boldsymbol{X}}{\partial y}\left(p_{0}\right)=\frac{\partial G}{\partial y}\left(p_{0}, \boldsymbol{\lambda}_{0}^{ \pm}\right)=0
$$

These imply that the lightcone $\mathfrak{g}_{\lambda_{0}^{ \pm}}^{-1}(0)=L C_{\lambda_{0}^{ \pm}}$is tangent to $M=\boldsymbol{X}(U)$ at $p_{0}=\boldsymbol{X}\left(x_{0}, y_{0}\right)$. In this case, we call each $L C_{\lambda_{0}^{ \pm}}$the tangent lightcone of $M=\boldsymbol{X}(U)$ at $p_{0}=\boldsymbol{X}\left(x_{0}, y_{0}\right)$.

We now describe the contacts of spacelike surfaces with lightcones. Let $L H_{M, i}^{\sigma}$ : $\left(U,\left(x_{i}, y_{i}\right)\right) \longrightarrow\left(L C_{+}^{*}, \boldsymbol{v}_{i}^{\sigma}\right), i=1,2$, be two lightlike hypersurface germs of spacelike surface germs $\boldsymbol{X}_{i}:\left(U,\left(x_{i}, y_{i}\right)\right) \longrightarrow\left(\mathbb{R}_{1}^{4}, p_{i}\right)$, where $\sigma= \pm$. We say that $L H_{M, 1}^{\sigma}$ and $L H_{M, 2}^{\sigma}$ are $\mathcal{A}$-equivalent if there exist diffeomorphism germs $\left.\phi:\left(U,\left(x_{1}, y_{1}\right)\right) \longrightarrow\left(U, x_{2}, y_{2}\right)\right)$ and $\Phi:\left(\mathbb{R}_{1}^{4}, \boldsymbol{\lambda}_{1}^{\sigma}\right) \longrightarrow\left(\mathbb{R}_{1}^{4}, \boldsymbol{\lambda}_{2}^{\sigma}\right)$ such that $\Phi \circ L H_{M, 1}^{\sigma}=L M_{M, 2}^{\sigma} \circ \phi$. If both of the regular sets of $L M_{M, i}^{\sigma}$ are dense in $\left(U,\left(x_{i}, y_{i}\right)\right)$, it follows from Proposition A.2 of the appendix that $L H_{M, 1}^{\sigma}$ and $L H_{M, 2}^{\sigma}$ are $\mathcal{A}$-equivalent if and only if the corresponding Legendrian lift germs are Legendrian equivalent. This condition is also equivalent to that two generating families $G_{1}$ and $G_{2}$ are $P$ - $\mathcal{K}$-equivalent by Theorem A.3, where $G_{i}:\left(U \times \mathbb{R}_{1}^{4},\left(\left(x_{i}, y_{i}\right), \boldsymbol{\lambda}_{i}^{\sigma}\right)\right) \longrightarrow \mathbb{R}$ denotes the Lorentzian distance-squared function germ of $\boldsymbol{X}_{i}$.

On the other hand, if we denote $g_{i, \lambda_{i}^{\sigma}}(x, y)=G_{i}\left(x, y, \boldsymbol{\lambda}_{i}^{\sigma}\right)$, then we have $g_{i, \lambda_{i}^{ \pm}}(x, y)=$ $\mathfrak{g}_{\lambda_{i}^{ \pm}} \circ \boldsymbol{x}_{i}(x, y)$. By Theorem 4.1, $K\left(\boldsymbol{X}_{1}(U), L C_{\lambda_{1}^{\sigma}}, \boldsymbol{\lambda}_{1}^{\sigma}\right)=K\left(\boldsymbol{x}_{2}(U), L C \lambda_{2}^{\sigma}, \boldsymbol{\lambda}_{2}^{\sigma}\right)$ if and only if 
$\widetilde{g}_{1, \lambda_{1}}$ and $\widetilde{g}_{2, \lambda_{2}}$ are $\mathcal{K}$-equivalent. Therefore, we can apply Proposition A.4 to our situation. We denote by $Q^{\sigma}\left(\boldsymbol{X},\left(x_{0}, y_{0}\right)\right)$ the local ring of the function germ $\widetilde{g}_{\lambda_{0}^{\sigma}}:\left(U,\left(x_{0}, y_{0}\right)\right) \longrightarrow \mathbb{R}$, where $\boldsymbol{\lambda}_{0}^{\sigma}=L C_{M}^{\sigma}\left(\left(x_{0}, y_{0}\right), u_{0}\right)$. We remark that we can explicitly write the local ring as follows:

$$
Q^{ \pm}\left(\boldsymbol{X},\left(x_{0}, y_{0}\right)\right)=\frac{C_{\left(x_{0}, y_{0}\right)}^{\infty}(U)}{\left\langle\left\langle\boldsymbol{X}(x, y), \widetilde{\boldsymbol{e}_{1} \pm \boldsymbol{e}_{2}}\left(x_{0}, y_{0}\right)\right\rangle-1\right\rangle_{C_{\left(x_{0}, y_{0}\right)}^{\infty}(U)}}
$$

where $C_{\left(x_{0}, y_{0}\right)}^{\infty}(U)$ is the local ring of function germs at $\left(x_{0}, y_{0}\right)$.

Theorem 4.3 Let $\boldsymbol{X}_{i}:\left(U,\left(x_{i}, y_{i}\right)\right) \longrightarrow\left(\mathbb{R}_{1}^{4}, \boldsymbol{X}_{i}\left(\left(x_{i}, y_{i}\right)\right)\right), i=1,2$, be spacelike surface germs such that the corresponding Legendrian lift germs are Legendrian stable. For $\sigma=$ + or - , the following conditions are equivalent:

(1) The lightlike hypersurface germs $L H_{M_{1}}^{\sigma}$ and $L H_{M_{2}}^{\sigma}$ are $\mathcal{A}$-equivalent.

(2) $G_{1}$ and $G_{2}$ are $P$-K $\mathcal{K}$-equivalent.

(3) $g_{1, \lambda_{1}}$ and $g_{2, \lambda_{2}}$ are $\mathcal{K}$-equivalent.

(4) $K\left(\boldsymbol{X}_{1}(U), L C_{\lambda_{1}^{\sigma}}, \boldsymbol{\lambda}_{1}^{\sigma}\right)=K\left(\boldsymbol{X}_{2}(U), L C \lambda_{2}^{\sigma}, \boldsymbol{\lambda}_{2}^{\sigma}\right)$.

(5) $Q^{\sigma}\left(\boldsymbol{X}_{1},\left(x_{1}, y_{1}\right)\right)$ and $Q^{\sigma}\left(\boldsymbol{X}_{2},\left(x_{2}, y_{2}\right)\right)$ are isomorphic as $\mathbb{R}$-algebras.

Proof. The preceding arguments shows that (3) and (4) are equivalent. The other assertions follow from Proposition A.4.

Given a spacelike surface germ $\boldsymbol{X}:\left(U,\left(x_{0}, y_{0}\right)\right) \longrightarrow\left(\mathbb{R}_{1}^{4}, \boldsymbol{X}\left(x_{0}, y_{0}\right)\right)$, we call

$$
\left(\boldsymbol{X}^{-1}\left(L C_{\lambda^{ \pm}}\right),\left(x_{0}, y_{0}\right)\right)
$$

the tangent lightcone indicatrix germ of $\boldsymbol{X}$, where $\boldsymbol{\lambda}^{ \pm}=\boldsymbol{X}\left(x_{0}, y_{0}\right)+u_{0}\left(\widetilde{\boldsymbol{e}_{1} \pm \boldsymbol{e}_{2}}\right)\left(x_{0}, y_{0}\right)$ and $u_{0}=-1 / \kappa_{i}^{\mp}\left(x_{0}, y_{0}\right), i=1,2$. As a corollary of Theorem 4.3 , we have

Corollary 4.4 Under the assumptions of Theorem 4.3, if the lightlike hypersurface germs $L H_{M_{1}}^{\sigma}$ and $L H_{M_{2}}^{\sigma}$ are $\mathcal{A}$-equivalent, then tangent lightcone indicatrix germs

$$
\left(\boldsymbol{X}_{1}^{-1}\left(L C_{\lambda_{1}^{ \pm}}\right),\left(x_{1}, y_{1}\right)\right) \quad \text { and } \quad\left(\boldsymbol{X}_{2}^{-1}\left(L C_{\lambda_{2}^{ \pm}}\right),\left(x_{2}, y_{2}\right)\right)
$$

are diffeomorphic as set germs.

Proof. Notice that the tangent lightcone indicatrix germ of $\boldsymbol{X}_{i}$ is the zero level set of $g_{i, \lambda_{i}}$. Since $\mathcal{K}$-equivalence among function germs preserves the zero-level sets of function germs, the assertion follows from Theorem 4.3. 


\section{Classification of singularities of lightlike hypersur- faces}

In this section we provide a generic classification of the singularities of lightlike hypersurfaces in $\mathbb{R}_{1}^{4}$. We consider the space of spacelike embeddings $\operatorname{Emb}_{\mathrm{sp}}\left(U, \mathbb{R}_{1}^{4}\right)$ with the Whitney $C^{\infty}$-topology. We also consider a function $\mathcal{G}: \mathbb{R}_{1}^{4} \times \mathbb{R}_{1}^{4} \longrightarrow \mathbb{R}$ defined by $\mathcal{G}(\boldsymbol{v}, \boldsymbol{\lambda})=\langle\boldsymbol{v}-\boldsymbol{\lambda}, \boldsymbol{v}-\boldsymbol{\lambda}\rangle$, and claim that $\mathcal{G}_{\lambda}$ is a submersion at $\boldsymbol{v} \neq \boldsymbol{\lambda}$ for any $\boldsymbol{\lambda} \in \mathbb{R}_{1}^{4}$, where $\mathcal{G}_{\lambda}(\boldsymbol{v})=\mathcal{G}(\boldsymbol{v}, \boldsymbol{\lambda})$. Given $\boldsymbol{X} \in \operatorname{Emb}_{\mathrm{sp}}\left(U, \mathbb{R}_{1}^{4}\right)$, we have $G=\mathcal{G} \circ\left(\boldsymbol{X} \times \mathrm{id}_{\mathbb{R}_{1}^{4}}\right)$. We also have the $\ell$-jet extension

$$
j_{1}^{\ell} G: U \times \mathbb{R}_{1}^{4} \longrightarrow J^{\ell}(U, \mathbb{R})
$$

defined by $j_{1}^{\ell} G(u, \boldsymbol{\lambda})=j^{\ell} g_{\lambda}(u)$, where we write $G(u, \boldsymbol{\lambda})=g_{\lambda}(u)$. Consider the trivialization $J^{\ell}(U, \mathbb{R}) \equiv U \times \mathbb{R} \times J^{\ell}(2,1)$. For any submanifold $Q \subset J^{\ell}(2,1)$, we denote $\widetilde{Q}=U \times\{0\} \times Q$. Then we have the following proposition as a corollary of Lemma 6 in Wassermann [18]. (See also Montaldi [16]).

Proposition 5.1 Let $Q$ be a submanifold of $J^{\ell}(n-1,1)$. Then the set

$$
T_{Q}=\left\{\boldsymbol{X} \in \operatorname{Emb}_{\mathrm{sp}}\left(U, \mathbb{R}_{1}^{4}\right) \mid j_{1}^{\ell} G \text { is transversal to } \widetilde{Q}\right\}
$$

is a residual subset of $\operatorname{Emb}_{\mathrm{sp}}\left(U, \mathbb{R}_{1}^{4}\right)$. If $Q$ is a closed subset, then $T_{Q}$ is open.

On the other hand, we have a stratification given by the set of $\mathcal{K}$-orbits in $J^{\ell}(2,1) \backslash$ $W^{\ell}(2,1)$ (For the definition of $W^{\ell}(2,1)$ and additional properties, refer to [5], Page 120). As a consequence of the above proposition, we have the following theorem.

Theorem 5.2 There exists an open dense subset $\mathcal{O} \subset \operatorname{Emb}_{\mathrm{sp}}\left(U, \mathbb{R}_{1}^{4}\right)$ such that for any $\boldsymbol{X} \in \mathcal{O}$, the germ of the Legendrian lift of the corresponding lightlike hypersurface $L H_{M}^{ \pm}$ at each point is Legendrian stable.

By the classification results on stable Legendrian mappings, we have the following

Corollary 5.3 There exists an open dense subset $\mathcal{O} \subset \operatorname{Emb}_{\mathrm{sp}}\left(U, \mathbb{R}_{1}^{4}\right)$ such that for any $\boldsymbol{X} \in \mathcal{O}$, the germ of the corresponding lightlike hypersurfaces $L H_{M}^{ \pm}$at any point $(x, y, u) \in$ $U \times \mathbb{R}$ is $\mathcal{A}$-equivalent to one of the map germs $A_{k}(1 \leq k \leq 4)$ or $D_{4}^{ \pm}$: where, $A_{k}, D_{4}^{ \pm}$-map germ $f:\left(\mathbb{R}^{3}, 0\right) \longrightarrow\left(\mathbb{R}^{4}, 0\right)$ are given by

$$
\begin{aligned}
& \left(A_{1}\right) f\left(u_{1}, u_{2}, u_{3}\right)=\left(u_{1}, u_{2}, u_{3}, 0\right), \\
& \left(A_{2}\right) f\left(u_{1}, u_{2}, u_{3}\right)=\left(3 u_{1}^{2}, 2 u_{1}^{3}, u_{2}, u_{3}\right), \\
& \left(A_{3}\right) f\left(u_{1}, u_{2}, u_{3}\right)=\left(4 u_{1}^{3}+2 u_{1} u_{2}, 3 u_{1}^{4}+u_{2} u_{1}^{2}, u_{2}, u_{3}\right), \\
& \left(A_{4}\right) f\left(u_{1}, u_{2}, u_{3}\right)=\left(5 u_{1}^{4}+3 u_{2} u_{1}^{2}+2 u_{1} u_{3}, 4 u_{1}^{5}+2 u_{2} u_{1}^{3}+u_{3} u_{1}^{2}, u_{1}, u_{2}\right), \\
& \left(D_{4}^{+}\right) f\left(u_{1}, u_{2}, u_{3}\right)=\left(2\left(u_{1}^{2}+u_{2}^{2}\right)+u_{1} u_{2} u_{3}, 3 u_{1}^{2}+u_{2} u_{3}, 3 u_{2}^{2}+u_{1} u_{3}, u_{3}\right), \\
& \left(D_{4}^{-}\right) f\left(u_{1}, u_{2}, u_{3}\right)=\left(2\left(u_{1}^{3}-u_{1} u_{2}^{2}\right)+\left(u_{1}^{2}+u_{2}^{2}\right) u_{3}, u_{2}^{2}-3 u_{1}^{2}-2 u_{1} u_{3}, u_{1} u_{2}-u_{2} u_{3}, u_{3}\right) .
\end{aligned}
$$


Proof. By Theorems 5.2 and A.3, the Lorentzian distance squared function $G$ is a $\mathcal{K}$ versal deformation of $g_{\lambda_{0}}$ at each $\left(x_{0}, y_{0}, \boldsymbol{\lambda}_{0}\right) \in U \times \mathbb{R}$. Therefore we can apply the generic classification of $\mathcal{K}$-versal deformations $F(x, y, \boldsymbol{\lambda})$ of function germs up to 4-parameters [1]. For any $F(x, y, \boldsymbol{\lambda})$, we define

$$
\Sigma_{*}(F)=\left\{(x, y, \boldsymbol{\lambda}) \mid F(x, y, \boldsymbol{\lambda})=\frac{\partial F}{\partial x}(x, y, \boldsymbol{\lambda})=\frac{\partial F}{\partial y}(x, y, \boldsymbol{\lambda})=0\right\}
$$

(cf. the appendix). The normal forms are given by

$$
\begin{aligned}
& F(x, y, \boldsymbol{\lambda})=x^{k+1} \pm y^{2}+\lambda_{1}+\lambda_{2} x+\cdots+\lambda_{k-1} x^{k-1}, 1 \leq k \leq 4 \\
& F(x, y, \boldsymbol{\lambda})=x^{3}+y^{3}+\lambda_{1}+\lambda_{2} x+\lambda_{3} y+\lambda_{4} x y \\
& F(x, y, \boldsymbol{\lambda})=x^{3}-x y^{2}+\lambda_{1}+\lambda_{2} x+\lambda_{3} y+\lambda_{4}\left(x^{2}+y^{2}\right) .
\end{aligned}
$$

For example, if we consider the germ given by

$$
F(x, y, \boldsymbol{\lambda})=x^{3}+y^{3}+\lambda_{1}+\lambda_{2} x+\lambda_{3} y+\lambda_{4} x y
$$

Then we get

$$
\Sigma_{*}(F)=\left\{\left(x, y, 2\left(x^{3}+y^{3}\right)+\lambda_{4} x y,-3 x^{2}-\lambda_{4} y,-3 y^{2}-\lambda_{4} x, \lambda_{4}\right) \mid\left(x, y, \lambda_{4}\right) \in \mathbb{R}^{3}\right\} .
$$

Therefore the corresponding Legendrian map germ is

$$
f\left(u_{1}, u_{2}, u_{3}\right)=\left(2\left(u_{1}^{2}+u_{2}^{2}\right)+u_{1} u_{2} u_{3}, 3 u_{1}^{2}+u_{2} u_{3}, 3 u_{2}^{2}+u_{1} u_{3}, u_{3}\right) \quad\left(D_{4}^{+}\right) .
$$

The other cases follow from similar arguments, so that we may leave the details to the readers.

By using the generic normal forms of generating families (i.e. Lorentzian distance squared functions) and Corollary 4.4, we have the following

Corollary 5.4 There exists an open dense subset $\mathcal{O} \subset \operatorname{Emb}_{\mathrm{sp}}\left(U, \mathbb{R}_{1}^{4}\right)$ such that for any $\boldsymbol{X} \in \mathcal{O}$, the germ of the corresponding tangent lightcone indicatrix at any point $\left(x_{0}, y_{0}\right) \in$ $U$ is diffeomorphic to one of the germs in the following list:

(1) $\left\{(x, y) \in\left(\mathbb{R}^{2}, 0\right) \mid x^{3}+y^{2}=0\right\}$ (ordinary cusp)

(2) $\left\{(x, y) \in\left(\mathbb{R}^{2}, 0\right) \mid x^{4} \pm y^{2}=0\right\}$ (tachnode or point)

(3) $\left\{(x, y) \in\left(\mathbb{R}^{2}, 0\right) \mid x^{5}+y^{2}=0\right\}$ (rhamphoid cusp)

(4) $\left\{(x, y) \in\left(\mathbb{R}^{2}, 0\right) \mid x^{3}-x y^{2}=0\right\}$ (three lines)

(5) $\left\{(x, y) \in\left(\mathbb{R}^{2}, 0\right) \mid x^{3}+y^{3}=0\right\}$ (line)

Proof. We have the same generic normal forms of generating families (i.e. Lorentzian distance squared function germs) at each point as in the above corollary. By Corollary 4.4, 
the corresponding lightcone tangent indicatrix germs are diffeomorphic to the zero-level set of the function germ $F \mid \mathbb{R}^{2} \times\{0\}$ of the list. For example, if the normal form is given by

$$
F(x, y, \boldsymbol{\lambda})=x^{3}+y^{3}+\lambda_{1}+\lambda_{2} x+\lambda_{3} y+\lambda_{4} x y,
$$

then we have $F \mid \mathbb{R}^{2} \times\{0\}=x^{3}+y^{3}$, so that the corresponding lightcone tangent indicatrix germ is diffeomorphic to the set germ (4) in the above list.

\section{The eikonal equation}

As indirect motivation we will show how the construction above is naturally encountered in solutions to the Minkowski eikonal equation:

$$
-\left(\frac{\partial S}{\partial x_{1}}\right)^{2}+\left(\frac{\partial S}{\partial x_{2}}\right)^{2}+\left(\frac{\partial S}{\partial x_{3}}\right)^{2}+\left(\frac{\partial S}{\partial x_{4}}\right)^{2}=0
$$

If the solution has a form $S\left(x_{1}, x_{2}, x_{3}, x_{4}\right)=x_{1}-U\left(x_{2}, x_{3}, x_{4}\right)$, we have a solution of the Euclidean eikonal equation:

$$
\left(\frac{\partial U}{\partial x_{2}}\right)^{2}+\left(\frac{\partial U}{\partial x_{3}}\right)^{2}+\left(\frac{\partial U}{\partial x_{4}}\right)^{2}=1
$$

The graph of the solution $U$ can be interpreted as a level set of $S$. If we consider a surface in Euclidean space as an initial manifold of the above Euclidean eikonal equation, we can obtain such a solution.

Let $\pi: T^{*}\left(\mathbb{R}_{1}^{4}\right) \longrightarrow \mathbb{R}_{1}^{4}$ be the cotangent bundle over $\mathbb{R}_{1}^{4}$ and $\left(\left(x_{1}, x_{2}, x_{3}, x_{4}\right),\left(p_{1}, p_{2}, p_{3}, p_{4}\right)\right)$ be the canonical coordinate system such that for a single valued solution $S$ we have $p_{i}=\partial S / \partial x_{i}$. Therefore the above eikonal equation can be viewed as a family of cones in $T^{*}\left(\mathbb{R}_{1}^{4}\right)$ given by the following equation:

$$
H\left(x_{1}, \boldsymbol{x}, p_{1}, \boldsymbol{p}\right)=\frac{1}{2}\left(-p_{1}^{2}+\boldsymbol{p} \cdot \boldsymbol{p}\right)=\frac{1}{2}\left(-p_{1}^{2}+p_{2}^{2}+p_{3}^{2}+p_{4}^{2}\right)=0
$$

where $\boldsymbol{x}=\left(x_{2}, x_{3}, x_{4}\right)$ and $\boldsymbol{p}=\left(p_{2}, p_{3}, p_{4}\right)$. The singularities of the hypersurface $H^{-1}(0)$ correspond to the zero section $\mathbb{R}_{1}^{4} \times\{\mathbf{0}\}$ of the cotangent bundle. Consider the 1 -form on $T^{*}\left(\mathbb{R}_{1}^{4}\right)$ given by

$$
\theta=-p_{1} d x_{1}+\boldsymbol{p} \cdot d \boldsymbol{x}
$$

where $\boldsymbol{p} \cdot d \boldsymbol{x}=\sum_{i=2}^{4} p_{i} d x_{i}$. We can show that $\theta \mid H^{-1}(0)$ is a contact form on the nonsingular part of $H^{-1}(0)$. If we consider a surface $\boldsymbol{X}(U)=M$ in Euclidean 3-space $\mathbb{R}^{3}=\{\boldsymbol{x}=$ $\left.\left(0, x_{2}, x_{3}, x_{4}\right) \mid \boldsymbol{x} \in \mathbb{R}^{4}\right\}$ and the unit normal vector $\boldsymbol{n}(x, y)$, then the surface $\boldsymbol{\ell}(x, y)=$ $(0, \boldsymbol{X}(x, y), 1, \boldsymbol{n}(x, y))$ in $T^{*} \mathbb{R}_{1}^{4}$ lies in the hypersurface $H^{-1}(0)$. Since $\boldsymbol{n}(x, y)$ is the normal 
vector of $M$, we have $\boldsymbol{\ell}^{*} \theta=\boldsymbol{n}(x, y) \cdot d \boldsymbol{X}(x, y)=0$. This means that the surface $\boldsymbol{\ell}(x, y)$ is an integral submanifold of $\theta \mid H^{-1}(0)$. Moreover, the Hamiltonian vector field along the surface $\boldsymbol{\ell}(x, y)$ is given by

$$
X_{H}=-\frac{\partial}{\partial x_{1}}+\boldsymbol{n}(x, y) \cdot \frac{\partial}{\partial \boldsymbol{x}} .
$$

It follows that we have a Cauchy problem for the level surface of a solution to the PDE $H\left(x_{1}, \boldsymbol{x}, p_{1}, \boldsymbol{p}\right)=0$ with the initial submanifold $\boldsymbol{\ell}(x, y)$. We can apply the characteristic method to obtain the level hypersurface of a multi-valued solution which is a Legendrian submanifold of $H^{-1}(0)$. In general, the level hypersurface of the solution to this Cauchy problem is the lightlike hypersurface. To see this, consider the 3-dimensional submanifold defined by

$$
L(x, y, u)=(u, \boldsymbol{X}(x, y)+u \boldsymbol{n}(x, y), 1, \boldsymbol{n}(x, y))
$$

in $T^{*} \mathbb{R}_{1}^{4}$. Since $\boldsymbol{n}(x, y)$ is a unit vector, we have $\boldsymbol{n}(x, y) \cdot d \boldsymbol{n}(x, y)=0$, so that

$$
L^{*} \theta=-d u+\boldsymbol{n}(x, y) \cdot d \boldsymbol{X}(x, y)+d u+\boldsymbol{n}(x, y) \cdot d \boldsymbol{n}(x, y)=0 .
$$

Therefore $L$ is a Legendrian embedding. It is clear that Image $L \subset H^{-1}(0)$. Moreover, if we set $\boldsymbol{e}_{1}(x, y)=(1,0,0,0)$ and $\boldsymbol{e}_{2}(x, y)=\boldsymbol{n}(x, y)$, then we have the lightlike hypersurface defined by

$$
L H_{M}^{ \pm}(x, y, u)=\boldsymbol{X}(x, y)+u\left(\boldsymbol{e}_{1} \pm \boldsymbol{e}_{2}\right)(x, y) .
$$

We remark that $\left(\widetilde{\boldsymbol{e}_{1} \pm \boldsymbol{e}_{2}}\right)(x, y)=\left(\boldsymbol{e}_{1} \pm \boldsymbol{e}_{2}\right)(x, y)$ in this case. Therefore, the above Legendrian embedding $L$ is the Legendrian lift of the lightlike hypersurface $L H_{M}^{ \pm}$. Since the simultaneity has no meanings in the theory of relativity, we might consider spacelike surfaces as initial submanifolds for the above Minkowski eikonal equation instead of surfaces in Euclidean space. Moreover, we have examples of lightlike hypersurface which cannot be constructed from a regular surface in $\mathbb{R}^{3}([9,10])$.

On the other hand, the Minkowski eikonal equation defines a hypersurface $H^{-1}(0) \times \mathbb{R}$ in the 1-jet space $J^{1}\left(\mathbb{R}_{1}^{4}, \mathbb{R}\right) \cong T^{*} \mathbb{R}_{1}^{4} \times \mathbb{R}$ on which the canonical contact structure is given by $d z-\theta$, where $(x, \boldsymbol{x}, p, \boldsymbol{p}, z)$ is the canonical coordinate system of $J^{1}\left(\mathbb{R}_{1}^{4}, \mathbb{R}\right)$. Under this framework, the Legendrian lift of each lightlike hypersurface in $H^{-1}(0)$ gives a non-characteristic initial data for the Cauchy problem of the Minkowski eikonal equation. Therefore we obtain the multivalued solution of the Cauchy problem by applying the characteristic method which is a Legendrian submanifold of $J^{1}\left(\mathbb{R}_{1}^{4}, \mathbb{R}\right)$ belonging to $H^{-1}(0) \times \mathbb{R}$. It follows that a general lightlike hypersurface can be considered as the level set of a multivalued solution of the Minkowski eikonal equation.

We have another interpretation as follows: Observe that there is a natural spherical blow up the 7-dimensional cone bundle $\{H=0\}$ in $T^{*} \mathbb{R}_{1}^{4}$ defined by

$$
\mathbb{R}_{1}^{4} \times \mathbb{R} \times S^{2} \longrightarrow T^{*} \mathbb{R}_{1}^{4}
$$


where $\left(x_{1}, \boldsymbol{x}, t, \theta\right) \longmapsto\left(x_{1}, \boldsymbol{x}, t(1, \theta)\right), t \in \mathbb{R}, \theta \in S^{2}$. The characteristic line field and the canonical 1-form $\theta$ pullback to the cylinder bundle with removable zero points. It follows that the Cauchy problem can be extended to the initial submanifold which intersects the zero section in $\{H=0\} \in T^{*} \mathbb{R}_{1}^{4}$. Moreover, there exist $C^{\infty}$-foliations of $\mathbb{R}^{3}$ with mild singularities which generate well posed initial data. For example, consider a foliation by level surfaces $f(\boldsymbol{x})=c$ possibly with critical points. Then the initial data

$$
\boldsymbol{x} \longrightarrow\left(0, \boldsymbol{x}, \sqrt{1-\left\|d f_{x}\right\|}, d f_{x}\right)
$$

will generate a 4-dimension submanifold in $\{H=0\}$ which is a family of multivalued 3-dimension Legendrian submanifolds in $\{H=0\}$ (i.e. a multivalued solution) on the complement of the critical points. For special cases of $f(\boldsymbol{x})=c$, this 4-manifold has a $C^{\infty}$-immersive extension to the missing points. In any case each nonsingular level surface $f(\boldsymbol{x})=c$ generates a lightlike hypersurface as in the above paragraph. These hypersurfaces are the "level 3-manifolds" of the multivalued solution.

\section{$7 \quad$ Lightlike hypersurface singularities in curved space- times}

Let $g$ denote a $C^{\infty}$-Lorentzian (pseudo) Riemannian metric on a neighbourhood of the origin in $\mathbb{R}^{4}$. We may choose local normal coordinates ([17], Proposition 33) so that the components $g_{i j}$ of $g$ satisfy

$$
g_{i j} \equiv \delta_{i j} \epsilon_{j} \bmod \mathcal{M}^{2}
$$

where $\epsilon_{1}=-1$ and $\epsilon_{j}=1, j \neq 1$. Recall that the conformal metric $c g, 0<c \in \mathbb{R}$ has the same unparametrized null geodesics as the original metric $g$. As in Section 2 the lightlike hypersurfaces of $g$ consist of two parameter families of null geodesics. It follows that a lightlike hypersurface for $c g$ is also lightlike for $g$. Hence via pullback over the dilation $d_{c}: \mathbb{R}^{4} \longrightarrow \mathbb{R}^{4}, \boldsymbol{x} \longmapsto 1 / \sqrt{c} \boldsymbol{x}$, for all $c>0$, we see that $g$ has the same lightlike hypersurface singularities (near the origin in $\mathbb{R}^{4}$ ) as the metric

$$
d_{c}^{*}\left(c g_{i j}\right)=\delta_{i j}+\frac{1}{c}(4 \text { th order terms }) .
$$

Thus, for sufficiently large $c$, we may use the generic nature of the results in Sections 4 and 5 to conclude that Corollary 5.3 is also valid for an open dense set of $C^{\infty}$ embeddings $U \longrightarrow$ $\left(\mathbb{R}^{4}, g\right)$. In other words, on a sufficiently small neighbourhood in any smooth Lorentzian 4-manifold, there exist stable lightlike hypersurface singularities as in Minkowski space. 


\section{Appendix Generating families}

Here we give a quick survey on the theory of Legendrian singularities mainly developed by Arnol'd-Zakalyukin $[1,19]$. Let $F:\left(\mathbb{R}^{k} \times \mathbb{R}^{n}, \mathbf{0}\right) \longrightarrow(\mathbb{R}, \mathbf{0})$ be a function germ. We say that $F$ is a Morse family if the map germ

$$
\Delta^{*} F=\left(F, \frac{\partial F}{\partial q_{1}}, \ldots, \frac{\partial F}{\partial q_{k}}\right):\left(\mathbb{R}^{k} \times \mathbb{R}^{n}, \mathbf{0}\right) \longrightarrow\left(\mathbb{R} \times \mathbb{R}^{k}, \mathbf{0}\right)
$$

is submersive, where $(q, x)=\left(q_{1}, \ldots, q_{k}, x_{1}, \ldots, x_{n}\right) \in\left(\mathbb{R}^{k} \times \mathbb{R}^{n}, \mathbf{0}\right)$. In this case we have a smooth $(n-1)$-dimensional submanifold

$$
\Sigma_{*}(F)=\left\{(q, x) \in\left(\mathbb{R}^{k} \times \mathbb{R}^{n}, \mathbf{0}\right) \mid F(q, x)=\frac{\partial F}{\partial q_{1}}(q, x)=\cdots=\frac{\partial F}{\partial q_{k}}(q, x)=0\right\}
$$

and the map germ $\Phi_{F}:\left(\Sigma_{*}(F), \mathbf{0}\right) \longrightarrow P T^{*} \mathbb{R}^{n}$ defined by

$$
\Phi_{F}(q, x)=\left(x,\left[\frac{\partial F}{\partial x_{1}}(q, x): \cdots: \frac{\partial F}{\partial x_{n}}(q, x)\right]\right)
$$

is a Legendrian immersion. Then we have the following fundamental theorem in the theory of Legendrian singularities ([1] §20.7 [19], Page 27).

Proposition A.1 All Legendrian submanifold germs in $P T^{*} \mathbb{R}^{n}$ are constructed by the above method.

We call $F$ a generating family of $\Phi_{F}$, and the corresponding wave front is $W\left(\Phi_{F}\right)=$ $\pi_{n}\left(\Sigma_{*}(F)\right)$, where $\pi_{n}: \mathbb{R}^{k} \times \mathbb{R}^{n} \longrightarrow \mathbb{R}^{n}$ is the canonical projection.

We now introduce an equivalence relation among Legendrian immersion germs. Let $i$ : $(L, p) \subset\left(P T^{*} \mathbb{R}^{n}, p\right)$ and $i^{\prime}:\left(L^{\prime}, p^{\prime}\right) \subset\left(P T^{*} \mathbb{R}^{n}, p^{\prime}\right)$ be Legendrian immersion germs. Then we say that $i$ and $i^{\prime}$ are Legendrian equivalent if there exists a contact diffeomorphism germ $H:\left(P T^{*} \mathbb{R}^{n}, p\right) \longrightarrow\left(P T^{*} \mathbb{R}^{n}, p^{\prime}\right)$ such that $H$ preserves fibers of $\pi$ and that $H(L)=L^{\prime}$. A Legendrian immersion germ into $P T^{*} \mathbb{R}^{n}$ at a point is said to be Legendrian stable if for every map with the given germ there is a neighbourhood in the space of Legendrian immersions (in the Whitney $C^{\infty}$ topology) and a neighbourhood of the original point such that each Legendrian immersion belonging to the first neighbourhood has in the second neighbourhood a point at which its germ is Legendrian equivalent to the original germ.

Since the Legendrian lift $i:(L, p) \subset\left(P T^{*} \mathbb{R}^{n}, p\right)$ is uniquely determined by the regular part of the wave front $W(i)$, we have the following simple but significant property of Legendrian immersion germs:

Proposition A.2 Let $i:(L, p) \subset\left(P T^{*} \mathbb{R}^{n}, p\right)$ and $i^{\prime}:\left(L^{\prime}, p^{\prime}\right) \subset\left(P T^{*} \mathbb{R}^{n}, p^{\prime}\right)$ be Legendrian immersion germs such that regular sets of $\pi \circ i$ and $\pi \circ i^{\prime}$ are dense respectively. Then $i, i^{\prime}$ are Legendrian equivalent if and only if wave front sets $W(i), W\left(i^{\prime}\right)$ are diffeomorphic as 
set germs. Here $\pi: P T^{*} \mathbb{R}^{n} \longrightarrow \mathbb{R}^{n}$ is the canonical projection of the projective cotangent bundle.

This result has been firstly pointed out by Zakalyukin ([20], Assertion 1.1). In his original assertion, he assume that the representatives of $\pi \circ i$ and $\pi \circ i^{\prime}$ are proper. However, we remark that we can get rid of such an assumption. The assumption in the above proposition is a generic condition for $i, i^{\prime}$. In particular, if $i$ and $i^{\prime}$ are Legendrian stable, then these satisfy the assumption.

We can interpret the Legendrian equivalence by using the notion of generating families. We denote by $\mathcal{E}_{n}$ the local ring of function germs $\left(\mathbb{R}^{n}, \mathbf{0}\right) \longrightarrow \mathbb{R}$ with the unique maximal ideal $\mathfrak{M}_{n}=\left\{h \in \mathcal{E}_{n} \mid h(0)=0\right\}$. Let $F, G:\left(\mathbb{R}^{k} \times \mathbb{R}^{n}, \mathbf{0}\right) \longrightarrow(\mathbb{R}, \mathbf{0})$ be function germs. We say that $F$ and $G$ are $P$ - $\mathcal{K}$-equivalent if there exists a diffeomorphism germ $\Psi:\left(\mathbb{R}^{k} \times \mathbb{R}^{n}, \mathbf{0}\right) \longrightarrow\left(\mathbb{R}^{k} \times \mathbb{R}^{n}, \mathbf{0}\right)$ of the form $\Psi(x, u)=\left(\psi_{1}(q, x), \psi_{2}(x)\right)$ for $(q, x) \in$ $\left(\mathbb{R}^{k} \times \mathbb{R}^{n}, \mathbf{0}\right)$ such that $\Psi^{*}\left(\langle F\rangle_{\mathcal{E}_{k+n}}\right)=\langle G\rangle_{\mathcal{E}_{k+n}}$. Here $\Psi^{*}: \mathcal{E}_{k+n} \longrightarrow \mathcal{E}_{k+n}$ is the pull back $\mathbb{R}$-algebra isomorphism defined by $\Psi^{*}(h)=h \circ \Psi$.

Let $F:\left(\mathbb{R}^{k} \times \mathbb{R}^{n}, \mathbf{0}\right) \longrightarrow(\mathbb{R}, \mathbf{0})$ be a function germ. We say that $F$ is a $\mathcal{K}$-versal deformation of $f=F \mid \mathbb{R}^{k} \times\{\mathbf{0}\}$ if

$$
\mathcal{E}_{k}=T_{e}(\mathcal{K})(f)+\left\langle\frac{\partial F}{\partial x_{1}}\left|\mathbb{R}^{k} \times\{\mathbf{0}\}, \ldots, \frac{\partial F}{\partial x_{n}}\right| \mathbb{R}^{k} \times\{\mathbf{0}\}\right\rangle_{\mathbb{R}},
$$

where

$$
T_{e}(\mathcal{K})(f)=\left\langle\frac{\partial f}{\partial q_{1}}, \ldots, \frac{\partial f}{\partial q_{k}}, f\right\rangle_{\mathcal{E}_{k}} .
$$

(See [12].) The main result in the theory ([1], §20.8 and [19], THEOREM 2) is the following:

Theorem A.3 Let $F, G:\left(\mathbb{R}^{k} \times \mathbb{R}^{n}, \mathbf{0}\right) \longrightarrow(\mathbb{R}, 0)$ be Morse families. Then

(1) $\Phi_{F}$ and $\Phi_{G}$ are Legendrian equivalent if and only if $F, G$ are $P$-K $\mathcal{K}$-equivalent, and

(2) $\Phi_{F}$ is Legendrian stable if and only if $F$ is a $\mathcal{K}$-versal deformation of $F \mid \mathbb{R}^{k} \times\{\mathbf{0}\}$.

Since $F$ and $G$ are function germs on the common space germ $\left(\mathbb{R}^{k} \times \mathbb{R}^{n}, \mathbf{0}\right)$, we do not need the notion of stably $P$ - $\mathcal{K}$-equivalences under this situation (cf. [19], Page 27). By the uniqueness result of the $\mathcal{K}$-versal deformation of a function germ, we have the following classification result of Legendrian stable germs (cf. [6]). For any map germ $f:\left(\mathbb{R}^{n}, \mathbf{0}\right) \longrightarrow\left(\mathbb{R}^{p}, \mathbf{0}\right)$, we define the local ring of $f$ by $Q(f)=\mathcal{E}_{n} / f^{*}\left(\mathfrak{M}_{p}\right) \mathcal{E}_{n}$.

Proposition A.4 Let $F$ and $G:\left(\mathbb{R}^{k} \times \mathbb{R}^{n}, \mathbf{0}\right) \longrightarrow(\mathbb{R}, 0)$ be Morse families. Suppose that $\Phi_{F}$ and $\Phi_{G}$ are Legendrian stable. The the following conditions are equivalent.

(1) $\left(W\left(\Phi_{F}\right), \mathbf{0}\right)$ and $\left(W\left(\Phi_{G}\right), \mathbf{0}\right)$ are diffeomorphic as germs.

(2) $\Phi_{F}$ and $\Phi_{G}$ are Legendrian equivalent. 
(3) $Q(f)$ and $Q(g)$ are isomorphic as $\mathbb{R}$-algebras,

where $f=F\left|\mathbb{R}^{k} \times\{\mathbf{0}\}, g=G\right| \mathbb{R}^{k} \times\{\mathbf{0}\}$.

\section{References}

[1] V. I. Arnol'd, S. M. Gusein-Zade and A. N. Varchenko, Singularities of Differentiable Maps vol. I. Birkhäuser, 1986.

[2] S. Chandrasekhar, The Mathematical Theory of Black Holes, International Series of Monographs on Physics. 69 Oxford Univeristy press, 1983.

[3] S. Frittelli, C. Kozameh and E. T. Newman, GR via characteristic surfaces, J. Math. Phys. 36 (1995), 4984-5004.

[4] S. Frittelli, E. T. Newman and G. Silva-Ortigoza, The Eikonal equation in flat space: Null surfaces and their singularities I, J. Math. Phys. 40 (1999), 383-407.

[5] C. G. Gibson, K. Wirthmuller, A. A. du Plessis and E. J. Looijenga, Topological stability of smooth mappings, Lecture Notes in Math. 552, Springer-Verlag, Berlin-New York,1976.

[6] S. Izumiya, D. Pei and T. Sano, The lightcone Gauss map and the lightcone developable of a spacelike curve in Minkowski 3-space, Glasgow. Math. J. 42 (2000), 75-89.

[7] S. Izumiya, D. Pei and M.C. Romero Fuster, The lightcone Gauss map of a spacelike surface in Minkowski 4-space, to appear in Asian J. Math.

[8] S. Izumiya, D. Pei and M.C. Romero Fuster, Umbilicity of space-like submanifolds of Minkowski space, Proc. Roy. Soc. Edinburh Sect. A 134 (2004), 375-387.

[9] M. Kossowski, The $S^{2}$-valued Gauss maps and split total cuvature of space-like codimension-2 surfaces in Minkowski space, J. London Math. Soc.(2) 40 (1989), 179192.

[10] M. Kossowski, The intrinsic conformal structure and Gauss map of a light-like hypersurface in Minkowski space, Trans. Amer. Math. Soc. 316 (1989), 369-383.

[11] J. A. Little, On singularities of submanifolds of high dimensional Euclidean space, Ann. Mat. Pura Appl.(4) 83 (1969), 261-335.

[12] J. Martinet, Singularities of Smooth Functions and Maps, London Math. Soc. Lecture Note Ser. 58, Cambridge Univ. Press, Cambridge-New York, 1982.

[13] J. N. Mather, Stability of $C^{\infty}{ }_{-}$mappings $I V$, Classification of stable germs by $\mathbb{R}$ algebras, Inst. Hautes Études Sci. Publ. Math. 37 (1970), 223-248.

[14] C. W. Misner, K. S. Thorpe and J. W. Wheeler, Gravitation, W. H. Freeman and Co., San Francisco, CA, 1973.

[15] J. A. Montaldi, On contact between submanifolds, Michigan Math. J. 33, (1986), 81-85. 
[16] J. A. Montaldi, On generic composites of maps, Bull. London Math. Soc. 23 (1991), $81-85$.

[17] B. O'Neill, Semi-Riemannian Geometry, Academic Press, New York, 1983.

[18] G. Wassermann, Stability of Caustics, Math. Ann. 210 (1975), 43-50.

[19] V. M. Zakalyukin, Lagrangian and Legendrian singularities, Funct. Anal. Appl. (1976), $23-31$.

[20] V. M. Zakalyukin, Reconstructions of fronts and caustics depending one parameter and versality of mappings, J. Sov. Math. 27 (1984), 2713-2735.

\section{SHYUiChi IZUMiYa}

Department of Mathematics

HOKKAIDO UNIVERSITY

SAPPORO 060-0810

JAPAN

E-mail address:izumiya@math.sci.hokudai.ac.jp

MareK Kossowski

Department of Mathematics

The University of South Carolina

Columbia Campus

Columbia

SC 29208

USA

E-mail address:kossowsk@math.sc.edu

Donghe Pei

Department of Mathematics

Northeast Normal University

ChangChun 130024

P.R.CHINA

E-mail address: donghepei@mail.jl.cn

María del Carmen Romero Fuster

Departament de Geometría I Topología

Universitat de VALÈnCia

46100 BuRJassot (VAlÈnCia)

ESPANYA

E-mail address: carmen.romero@post.uv.es 